\title{
Pigeons concurrently categorize photographs at both basic and superordinate levels
}

\author{
OLGA F. LAZAREVA, KATE L. FREIBURGER, and EDWARD A. WASSERMAN \\ University of Iowa, Iowa City, Iowa
}

\begin{abstract}
We studied categorization in pigeons, using carefully controlled photographs. Within daily sessions, 4 pigeons had to classify each of 32 photographs into either its proper basic-level category (cars, chairs, flowers, or people; four-key forced choice procedure) or its proper superordinate-level category (natural or artificial; two-key forced choice procedure). The pigeons successfully classified the same stimuli at both levels. Overall, the pigeons learned the basic discrimination more quickly than the superordinate discrimination, but this difference was reliable only for artificial stimuli (cars and chairs), not for natural stimuli (flowers and people). The pigeons also exhibited reliable discrimination transfer to novel photographs, attesting to the open-endedness of these basic and superordinate categories.
\end{abstract}

Categorizing environmental stimuli and generalizing these categories to novel instances are fundamental to many human cognitive activities. Some categories have a strict definition: A circle contains the set of all points that are equidistant from a reference point. But many categories do not have such clear-cut definitions. Instead of an object either belonging to a category or not, an object may differentially exemplify that category (E. E. Smith \& Medin, 1981). Considerable research has been directed at understanding object categorization in humans (reviewed by Hampton, 2001; Mareschal \& Quinn, 2001).

\section{Experimental Study of Object Categorization in Animals}

Object categorization has also been studied in nonhuman animals. Such studies typically involve animals' discrimination of photographs of objects from human language categories, using a variety of behavioral paradigms. In the first investigation of object categorization by birds, Herrnstein and Loveland (1964) trained pigeons to peck if a photograph contained a person and to refrain from pecking if a photograph did not contain a person. The pigeons readily learned the task and transferred the discrimination to novel photographs. Subsequent researchers have found that several animal species can acquire this presencel absence discrimination involving pictures of people, trees, fish, or conspecifics (Aust \& Huber, 2001; D'Amato \& Van Sant, 1988; Poole \& Lander, 1971; Sands, Lincoln, \&

This research was supported by National Institute of Mental Health Grant MH47313. We thank Jason Bechen and Maureen Freiburger for help in photographing the person models and Maureen and Wayne Freiburger, Jake Freiburger and friends, Jason Bechen, Sandy and John Bechen, and the Great River Fiber Artists for being such willing models. We also thank Lisa Oakes and Brian Ross for their helpful comments on an early version of the manuscript. Correspondence concerning this article should be addressed to O. F. Lazareva, E11 Seashore Hall, Department of Psychology, University of Iowa, Iowa City, IA 52242 (e-mail: olga-lazareva@uiowa.edu).
Wright, 1982; Schrier \& Brady, 1987), as well as generalize this discrimination to novel images.

But most human language categories are not of the presence/absence sort (Herrnstein, 1984; Sands et al., 1982); instead, humans carve the world into multiple clusters of related objects, like rocks and birds. Starting from this more realistic multiple categorization scheme, Bhatt, Wasserman, Reynolds, and Knauss (1988) found that pigeons reliably categorized both old and new examples of cats, flowers, cars, and chairs by pecking one of four different buttons. Thus, pigeons can learn at least four noncomplementary and open-ended categories of real-world visual stimuli.

\section{Basic and Superordinate Categorization in Humans and Nonhuman Animals}

The previously cited reports concerned object categorization at the basic level. Basic-level categories are commonly contrasted with superordinate categories, which comprise several perceptually diverse basic-level categories. Unlike basic categories, superordinate categories are not believed to be based on perceptual similarity among their members (Hampton, 2001).

In a seminal paper, Rosch, Mervis, Gray, Johnson, and Boyes-Braem (1976) found that children learn to classify images at the basic level more quickly and at an earlier age than at the superordinate level. Later investigations showed, however, that infants may be sensitive to broad global categories (e.g., vehicles vs. animals), but not to narrower basic-level categories within the superordinate category (e.g., rabbit vs. dog or car vs. track; Mandler \& McDonough, 2000). Some researchers have suggested that whether the infant will selectively attend at the global or the basic level may depend on the context of the task (Mareschal \& Quinn, 2001; Ribar, Oakes, \& Spalding, 2004).

The findings on human categorization lead to the following question: Can nonhuman animals also learn super- 
ordinate discriminations? The answer is yes. Nonhuman animals, including pigeons, learn to respond similarly to pictures from different superordinate-level categories (Roberts \& Mazmanian, 1988; Vonk \& McDonald, 2002; Wasserman, DeVolder, \& Coppage, 1992). For example, Wasserman et al. trained pigeons to peck one button when photographs of either flowers or people (natural stimuli) were presented and to peck a second button when photographs of either cars or chairs (artificial stimuli) were presented. Then, with the first two buttons unavailable, they trained the pigeons to peck a third button to photographs of flowers and to peck a fourth button to photographs of cars. Finally, they showed the pigeons photographs of people and chairs with only the third and fourth buttons available. The pigeons predominately pecked the third button to photographs of people and the fourth button to photographs of chairs, attesting to the pigeons' having learned the superordinate categories of flowers plus people and cars plus chairs.

So even pigeons can categorize photographs at both basic and superordinate levels when each discrimination task is given alone. But humans flexibly discriminate the same stimulus at multiple levels of categorization, referring to a particular object as a Cadillac, a car, a vehicle, or an artifact. Flexible classification of objects and events at different levels is thought to be one of the most important and perhaps unique features of human categorization (Markman, 1989). Can animals flexibly classify the same stimulus at both basic and superordinate levels, depending on task demands? And if they can, might they differentially learn the basic and the superordinate discriminations? To date, we know of no single study of nonhuman animals that has involved the concurrent training of both basic and superordinate categorization of the same stimuli to answer these questions. That was the prime aim of the present experiment.

\section{Present Experiment}

We used eight stimuli from each of four basic-level categories - cars, chairs, flowers, and people - that are discriminable by pigeons (Astley \& Wasserman, 1992). The stimuli were colored photos of a single target object on a solid gray background to control for inadvertent cues (Edwards \& Honig, 1987; Greene, 1983), such as the area, orientation, and dominant color of the target image. From these four basic-level categories, we arranged two superordinate categories: natural stimuli (flowers plus people) and artificial stimuli (cars plus chairs).

During training, each photograph randomly required discrimination at both the basic and the superordinate levels. For example, if a photograph of a car was shown along with four choice keys, the pigeon was required to select the key that was associated with all of the car stimuli. Alternately, if a photograph of the car was shown along with two other choice keys, the pigeon was required to select the key that was associated with all of the artificial stimuli (cars and chairs). Both types of categorization trials occurred equally often during training.
Our pigeons successfully mastered both discrimination tasks, thus exhibiting the ability to categorize the photos in a flexible way. The birds also mastered the basic-level discrimination more quickly, on average, than they mastered the superordinate discrimination; detailed analysis revealed that only artificial stimuli (cars and chairs) were discriminated more readily at the basic level than at the superordinate level. Finally, after the pigeons had mastered both tasks, we showed them eight novel stimuli from each of the four basic-level categories. The birds exhibited similar and reliable transfer to novel photographs at both levels of categorization, although performance was reliably lower to the testing images than to the training images. These combined results suggest that pigeons can flexibly discriminate the same photographs, having apparently formed open-ended, noncomplementary classes of stimuli at both levels of categorization.

\section{METHOD}

\section{Subjects}

Four experimentally naive feral pigeons (Columba livia) were maintained at $85 \%$ of their free-feeding body weights by controlled daily feeding.

\section{Stimuli}

The 64 discriminative stimuli were photographs from the WorldWide Web plus digital photographs taken by the second author. The original background of each photograph was replaced by a solid $20 \%$ gray shading, using Photoshop 7.0 and KnockOut 2 (Procreate, Ottawa). The stimuli came from four basic-level categories: cars, chairs, flowers, and people. We created eight subsets of photographs composed of 2 stimuli in each category that were primarily black, blue, green, orange, pink, purple, red, or yellow. Small areas of other colors (such as the tires of cars, the faces of people, and the legs of chairs) were allowed as well. All of the target objects had approximately the same area and were presented in different orientations counterbalanced across images. The 64 images were divided into two sets ( 1 and 2 ) of 32 whose colors were balanced across black, blue, green, orange, pink, purple, red, or yellow. Figure 1 shows 4 training stimuli, one from each basic-level category.

\section{Apparatus}

Four $36 \times 36 \times 41 \mathrm{~cm}$ operant-conditioning chambers were used and placed in a room with continuous white noise. The photographs were presented on a 15-in. LCD monitor located behind an AccuTouch resistive touchscreen (Elo TouchSystems, Fremont, CA). Each chamber was controlled by an Apple iMac computer. The experimental procedure was programmed in HyperCard, Version 2.4.

One 10.16-cm square, or button, in the middle of the screen was used to display the photographs and to record the observing of pecks; the rest of the screen was black. Six black Macintosh icons on white backgrounds served as the report buttons and were $3.30 \mathrm{~cm}$ wide $\times 2.79 \mathrm{~cm}$ high. The basic-level report buttons were placed at the corners of the square center button. For 2 birds, the superordinatelevel buttons were above and below the central display; for the other 2 birds, the superordinate-level buttons were left and right of the central display.

\section{Behavioral Measures}

Because two- and four-alternative choice tasks involve different levels of chance performance ( $50 \%$ and $25 \%$, respectively), direct comparison of accuracy scores was inappropriate. So we transformed the percentage of correct choices to the signal detection 

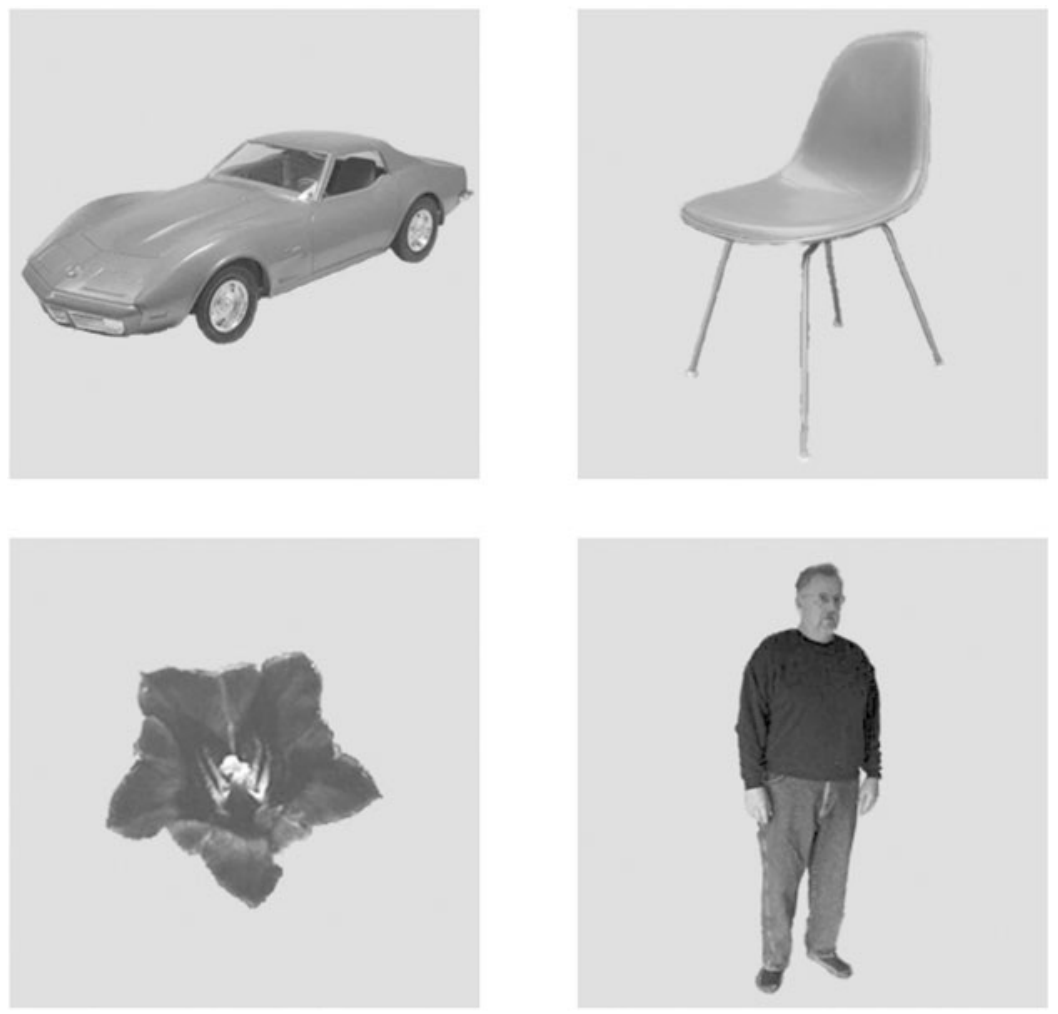

Figure 1. A set of 4 of the 64 stimuli that were chosen from four basic-level categories: cars, chairs, flowers, and people. The complete collection of training and testing stimuli can be seen in color at http://www.psychology.uiowa.edu/Faculty/Wasserman/.

measure $d^{\prime}$ (Algorithm 1; J. E. K. Smith, 1982). Signal detection theory has been used to interpret performance in several perceptual and cognitive tasks, and $d^{\prime}$ can be calculated for many measurement techniques and psychophysical procedures, including percentage correct and forced choice procedures (Green \& Swets, 1966). The chance level of discrimination corresponded to a $d^{\prime}$ of 0.00 for both tasks. For all statistical tests, alpha was set at .05.

\section{Procedure}

Training. During training, the pigeons' task could be either a four-alternative forced choice report of the basic categories or a two-alternative forced choice report of the superordinate categories. A trial began with the pigeon being shown a black cross in the center of the white display screen. Following one peck anywhere on the display, a training photograph appeared. The bird had to complete an observing response requirement (10-45 pecks for different pigeons) to the stimulus; then the report buttons appeared. On a basic trial, four report buttons were presented; on a superordinate trial, two different report buttons were shown. Basic and superordinate trials were randomly presented throughout a session. For 2 pigeons, the training stimuli came from Set 1; for the other two pigeons, the training stimuli came from Set 2.

If the pigeon's report response was correct, food was delivered, and the intertrial interval ensued. If the pigeon's report response was incorrect, the houselight and the monitor screen darkened, and a correction trial was given. Correction trials continued to be given until the correct response was made. All the responses were recorded, but only the first report response of each trial was scored in data analysis.

We planned to train all of the pigeons until they reached the 78/89 criterion $(78 \%$ correct to each basic category and $89 \%$ correct to each superordinate category) in a single session, so that both percentages yielded equal $d^{\prime}$ s of 1.8 . But only 1 bird reached this criterion in a timely fashion ( 21 sessions); the other 3 birds failed to reach this criterion in 70 training sessions. So the criterion level for these 3 pigeons was slightly lowered to $d^{\prime}$ 's of 1.7 , which corresponded to $75 \%$ correct for each basic category and $88 \%$ correct for each superordinate category. The selected criterion had to be maintained during testing; if a pigeon's discrimination performance fell below this level, it was returned to training until it again reached criterion.

Testing. In testing, a novel set of 32 images was shown. Each testing session comprised 144 trials, with a ratio of training to testing stimuli of 8:1. Testing lasted 12 days, so that each novel stimulus was shown three times in the basic task and three times in the superordinate task. The pigeons trained with Set 1 stimuli were tested with Set 2 stimuli; pigeons trained with Set 2 stimuli were tested with Set 1 stimuli. For the training stimuli, the pigeons received reinforcement only after a correct response; incorrect responses led to one or more correction trials. For the testing stimuli, the birds received reinforcement after all responses.

\section{RESULTS}

\section{Acquisition of Basic and Superordinate Categorization Tasks}

Training to criterion took a mean of $59.3 \pm 12.8$ days, with a minimum of 21 days and a maximum of 73 days. To analyze acquisition, we calculated the number of sessions required to reach $d^{\prime}$ s of $0.0,0.3,0.6,0.9,1.2,1.5$, and 1.8. A $d^{\prime}$ of 0.0 corresponded to chance; a $d^{\prime}$ of 1.8 
was the highest level that all the birds met to individual basic and superordinate categories.

Figure 2A shows that fewer sessions were needed to reach each criterion for the basic task than for the superordinate task, suggesting that the pigeons found the basic task easier than the superordinate task. This analysis did not consider the fact that two of the four basic categories were artificial objects and the other two basic categories were natural objects. So, we combined the $d^{\prime}$ scores from the flower and the person basic trials to create a score for the basic natural categories, and we combined the $d^{\prime}$ scores from the car and the chair basic trials to create a score for the basic artificial categories. We then compared these scores with the pigeons' responding to the superordinate natural category and the superordinate artificial category.

Figure 2B shows that the artificial stimuli (cars and chairs) were more readily classified at the basic level than at the superordinate level. But the speed of learning was similar for the natural stimuli (flowers and people) at both levels; indeed, the superordinate task was learned slightly

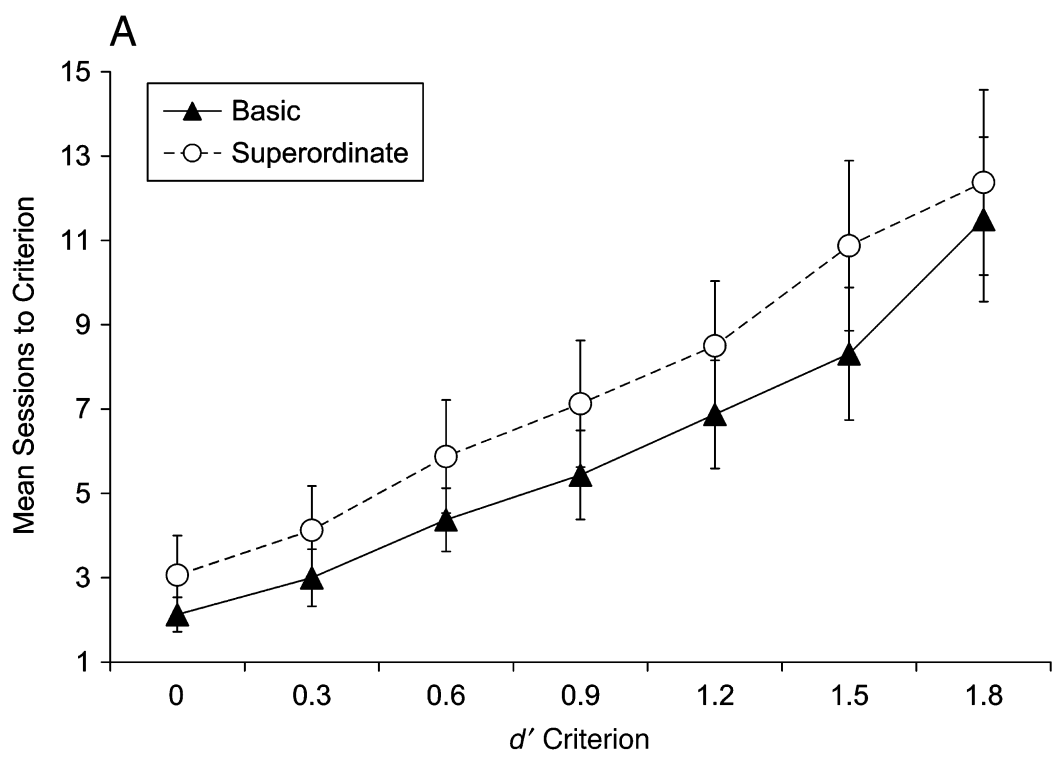

B

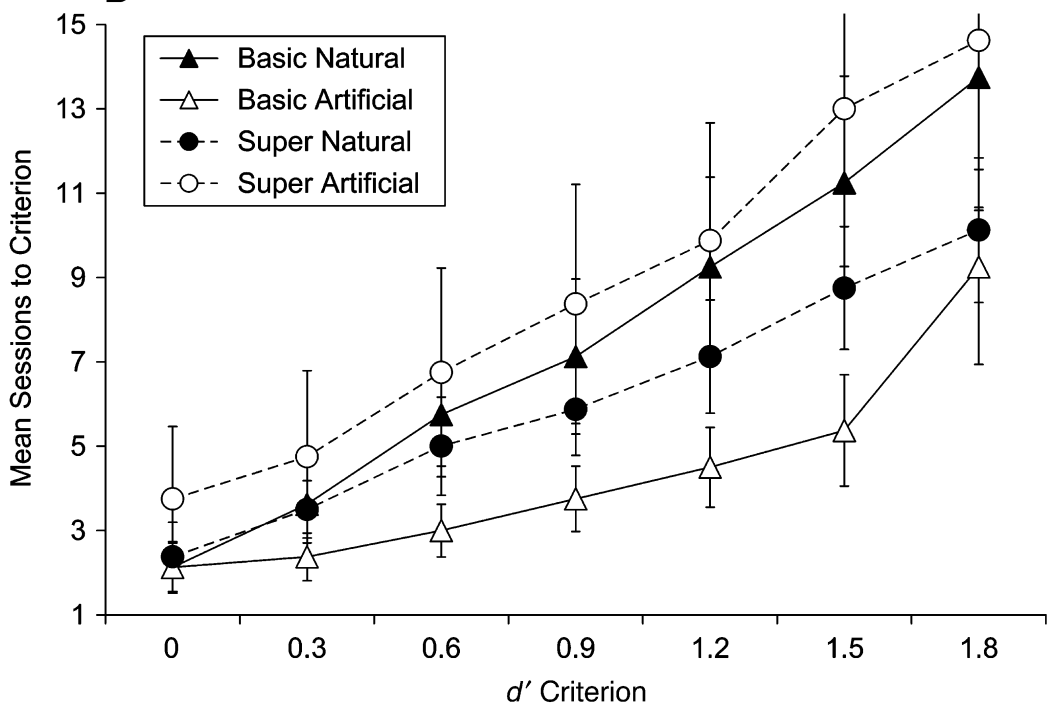

Figure 2. Mean number of sessions required to reach $d^{\prime}$ criterion throughout training on (A) basic and superordinate tasks and (B) basic artificial, basic natural, superordinate artificial, and superordinate natural tasks. A $d^{\prime} \mathbf{0}$ of corresponds to $25.0 \%$ and $50.0 \%$, a $d^{\prime}$ of 0.3 corresponds to $33.1 \%$ and $58.7 \%$, a $d^{\prime}$ of 0.6 corresponds to $42.2 \%$ and $66.8 \%$, a $d^{\prime}$ of 0.9 corresponds to $51.9 \%$ and $74.1 \%$, a $d^{\prime}$ of 1.2 corresponds to $61.5 \%$ and $80.2 \%$, a $d^{\prime}$ of 1.5 corresponds to $70.3 \%$ and $85.2 \%$, and a $d^{\prime}$ of 1.8 corresponds to $77.8 \%$ and $89.1 \%$, for basic and superordinate tasks, respectively. 
more quickly than the basic task. This pattern of results was true for each of the four basic categories within the natural and artificial groups (not depicted in Figure 2).

An analysis of variance (ANOVA) with task (basic or superordinate), category (natural or artificial), and criterion (7) as factors and with mean sessions to criterion as the dependent measure revealed a significant main effect of criterion $[F(6,3)=11.56]$, but no significant effect of category $[F(1,3)=0.16]$. Of course, the number of sessions to criterion necessarily increased with the increase in the $d^{\prime}$ criterion, making this main effect nondiagnostic. More important, the ANOVA revealed a significant main effect of task $[F(1,3)=3.98]$, indicating that, overall, the basic categorization task was mastered more quickly than the superordinate categorization task. The ANOVA also showed a significant task $\times$ category interaction $[F(1,1)=$ 15.65]. The basic artificial task was mastered more slowly than the superordinate artificial task (4.34 and 8.73 sessions, respectively), a reliable difference; the natural basic task was mastered slower than natural superordinate task (7.55 and 6.11 sessions, respectively), but this difference was not reliable. No other interactions were significant.

\section{Natural and Artificial Objects}

Our pigeons classified the artificial stimuli more quickly at the basic than at the superordinate level, but this was not true for the natural stimuli. This was an intriguing and unexpected finding, because there is no clear evidence of any difference in animals' categorizing of natural and artificial stimuli. Early reports suggested that pigeons might be unable to categorize artificial stimuli (Herrnstein, 1984); however, later studies showed no difference in pigeons' ability to classify images of natural or artificial stimuli (e.g., Bhatt et al., 1988).

One might explain our findings by hypothesizing that there was greater similarity between the chosen exemplars of natural objects than between the chosen exemplars of artificial objects. Highly dissimilar exemplars of artificial categories may be easy to distinguish at the basic level but hard to group at the superordinate level, whereas relatively similar exemplars of natural categories may be easy to group at the superordinate level but difficult to distinguish at the basic level. This pattern of similarity also suggests that the pigeons should more readily confuse flowers with people than with chairs or cars. In fact, over the entirety of basic-level training, the mean percentage of confusion errors for the natural stimuli $(47.0 \%)$ was significantly higher than chance $(33.3 \%$; one out of three keys represented a confusion error) by binomial test, whereas the mean percentage of confusion errors did not differ significantly from chance for the artificial stimuli $(30.47 \%)$. These results suggest that the members of the two natural categories did resemble one another more than the members of the two artificial categories did and that this difference in between-category similarity might explain the difference in acquisition of categorization at the two different levels for the natural and the artificial stimuli.

\section{Transfer of Basic and Superordinate Categorization to Novel Stimuli}

Figure 3 shows the results of transfer testing to the eight novel photographs in each of the four basic categories. The pigeons accurately transferred discriminative responding to the novel photographs. They showed similar stimulus generalization in each of the two tasks (Figure $3 \mathrm{~A}$ ) and to both the natural and the artificial stimuli (Figure 3B).

Binomial tests disclosed that transfer to the novel stimuli in both basic and superordinate categories differed significantly from chance. An ANOVA with task (basic or superordinate), category (natural or artificial), and trial type (training or testing) as factors and with $d^{\prime}$ as the dependent variable yielded significant main effects of task $[F(1,3)=4.4]$ and trial type $[F(1,3)=66.71]$, but no significant main effect of category $[F(1,3)=$ $2.87, p=.09]$ and no significant interactions $(F \mathrm{~s}<0.5)$. In other words, average training and testing performance on the basic categorization task was higher than that on the superordinate categorization task. The pigeons discriminated the novel natural and artificial testing stimuli at similar levels of accuracy, but they did so at lower levels than they discriminated the familiar training stimuli.

\section{DISCUSSION}

\section{Pigeons are Flexible Categorizers}

We found that pigeons can concurrently categorize the same photographs at both basic (car, chair, flower, or person) and superordinate (natural or artificial) levels. To our knowledge, this is the first evidence that nonhuman animals can flexibly classify the same visual stimuli at different categorization levels, depending on task demands. Flexible categorization appears not to be a uniquely human ability.

\section{Pigeons More Readily Categorize at the Basic Level Than at the Superordinate Level}

Our pigeons more quickly mastered the basic than the superordinate categorization task on average, despite the fact that the same photographic stimuli were used in each. To our knowledge, this is the first evidence that nonhuman animals may more readily classify photographs at the basic than at the superordinate level. However, detailed analysis of the data revealed that this trend was true only for artificial stimuli (cars and chairs), but not for natural stimuli (flowers and people). The pigeons' confusion errors suggested that flowers and people are perceptually more similar to each other than to either cars or chairs, whereas cars and chairs are just as similar to one another as they are to either flowers or people.

\section{Pigeons Similarly Transfer Basic-Level and Superordinate-Level Discriminations to Novel Stimuli}

After our pigeons had mastered both categorization tasks, they reliably transferred discriminative respond- 

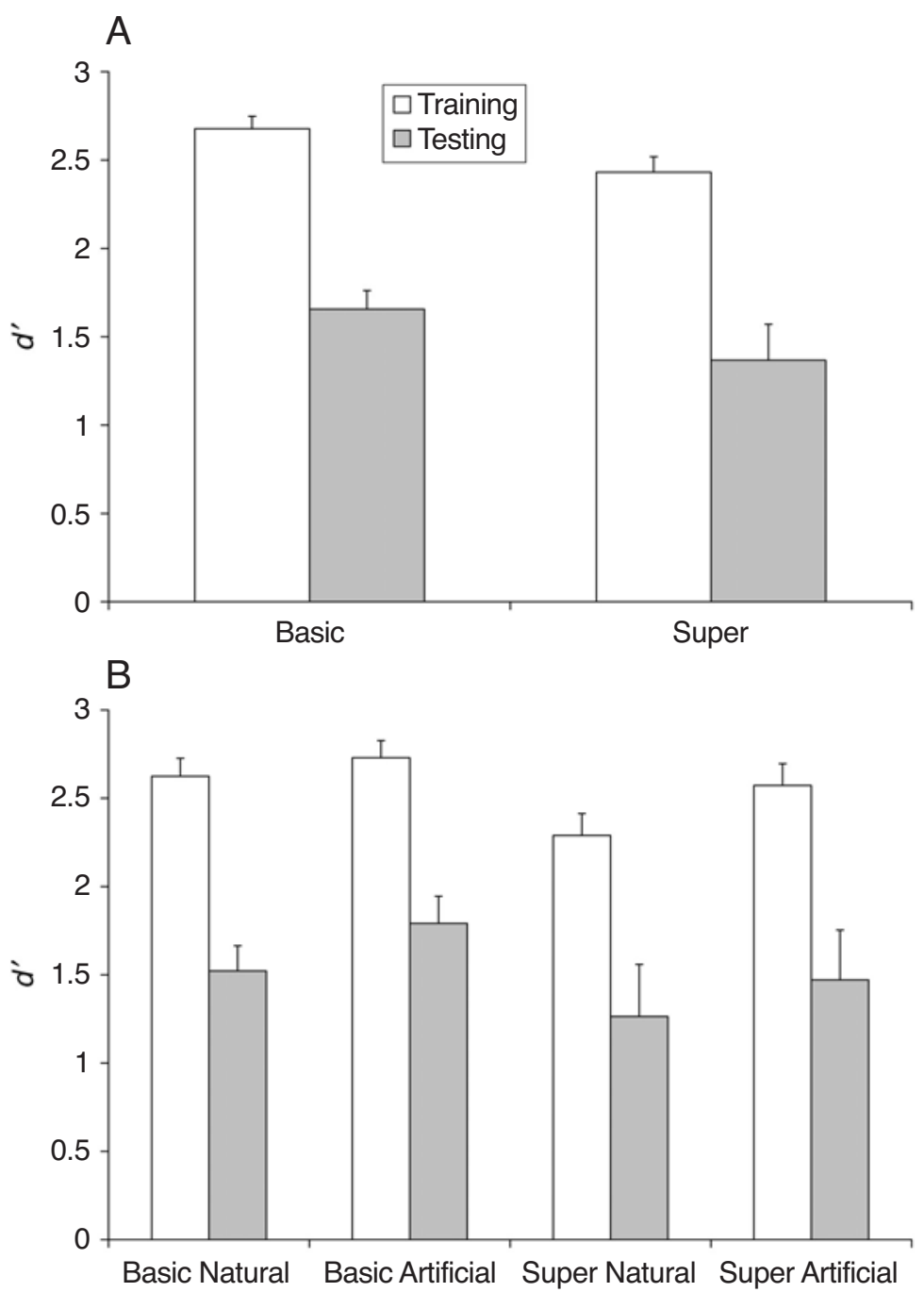

Figure 3. Mean discrimination performance during testing sessions. (A) Responding to the training and testing stimuli on basic and superordinate trials. (B) Responding to natural and artificial training and testing stimuli on basic and superordinate trials. Mean accuracy on the training trials was $89.49 \%$ and $\mathbf{9 2 . 2 9 \%}$ on the basic and the superordinate trials, respectively. Mean accuracy on the testing trials was $68.49 \%$ and $70.83 \%$ on the basic and the superordinate trials, respectively.

ing to novel exemplars at similar levels of accuracy; such discrimination transfer is the hallmark of conceptual behavior. In neither case was transfer accuracy to novel exemplars as high as it was to familiar exemplars, consistent with earlier research suggesting memorization of some or all of the individual training stimuli (Bhatt et al., 1988; Greene, 1983). Our pigeons similarly transferred their discriminations of natural and artificial stimuli.

\section{Conclusions}

Our data show that pigeons can concurrently categorize the same photographs at both basic (cars, chairs, flowers, and people) and superordinate (natural and artificial) levels, although they learn the basic task more quickly than the superordinate task. As well, pigeons can sort novel photographs into either basic or superordinate categories, depending on task demands, albeit at a lower level of accuracy than they sort familiar photographs. Unexpectedly, our pigeons showed differential responding to natural and artificial stimuli, a result that that has not been reported in previous studies (e.g., Astley \& Wasserman, 1992). Future research will explore the features of the photographic stimuli that control the pigeons' discrimination behavior in both basic and superordinate categorization tasks, with a keen eye toward any differences in control that may emerge in different tasks and for different types of stimuli (Aust \& Huber, 2001; Lubow, 1974). 


\section{REFERENCES}

Astley, S. L., \& Wasserman, E. A. (1992). Categorical discrimination and generalization in pigeons: All negative stimuli are not created equal. Journal of Experimental Psychology: Animal Behavior Processes, 18, 193-207.

AUST, U., \& HUBER, L. (2001). The role of item- and category-specific information in the discrimination of people versus nonpeople images by pigeons. Animal Learning \& Behavior, 29, 107-119.

Bhatt, R. S., Wasserman, E. A., Reynolds, W. F., Jr., \& Knauss, K. S. (1988). Conceptual behavior in pigeons: Categorization of both familiar and novel examples from four classes of natural and artificial stimuli. Journal of Experimental Psychology: Animal Behavior Processes, 14, 219-234.

D'Amato, M. R., \& VAN SANT, P. (1988). The person concept in monkeys (Cebus apella). Journal of Experimental Psychology: Animal Behavior Processes, 14, 43-55.

EDWARDS, C. A., \& HoNIG, W. K. (1987). Memorization and "feature selection" in the acquisition of natural concepts in pigeons. Learning \& Motivation, 18, 235-260.

Green, D. M., \& Swets, J. A. (1966). Signal detection theory and psychophysics. New York: Wiley.

Greene, S. L. (1983). Feature memorization in pigeon concept formation. In M. L. Commons, R. J. Herrnstein, \& A. R. Wagner (Eds.), Quantitative analyses of behavior: Vol. 4. Discrimination processes (pp. 209-231). Cambridge, MA: Ballinger.

HAMPTON, J. A. (2001). The role of similarity in natural categorization. In U. Hahn \& M. Ramscar (Eds.), Similarity and categorization (pp. 13-28). Oxford: Oxford University Press.

Herrnstein, R. J. (1984). Objects, categories, and discriminative stimuli. In H. L. Roitblat, T. C. Bever, \& H. S. Terrace (Eds.), Animal cognition (pp. 233-261). Hillsdale, NJ: Erlbaum.

Herrnstein, R. J., \& Loveland, D. H. (1964). Complex visual concept in the pigeon. Science, 146, 549-551.

Lubow, R. E. (1974). High-order concept formation in the pigeon. Journal of the Experimental Analysis of Behavior, 21, 475-483.

MANDLER, J. M., \& MCDonough, L. (2000). Advancing downward to the basic level. Journal of Cognition \& Development, 1, 379-403.
Mareschal, D., \& Quinn, P. C. (2001). Categorization in infancy. Trends in Cognitive Sciences, 5, 443-450.

Markman, E. M. (1989). Categorization and naming in children: Problems of induction. Cambridge, MA: MIT Press.

Poole, J., \& LANDER, D. G. (1971). The pigeon's concept of pigeon. Psychonomic Science, 25, 157-158.

Ribar, R. J., OAKes, L. M., \& Spalding, T. L. (2004). Infants can rapidly form new categorical representations. Psychonomic Bulletin \& Review, 11, 536-541.

Roberts, W. A., \& Mazmanian, D. S. (1988). Concept learning at different levels of abstraction by pigeons, monkeys, and people. Journal of Experimental Psychology: Animal Behavior Processes, 14, 247-260.

Rosch, E., Mervis, C. B., Gray, W. D., Johnson, D. M., \& BoyesBraEm, P. (1976). Basic objects in natural categories. Cognitive Psychology, 8, 382-439.

Sands, S. F., LinCOLN, C. E., \& Wright, A. A. (1982). Pictorial similarity judgments and the organization of visual memory in the rhesus monkey. Journal of Experimental Psychology: General, 111, 369389.

Schrier, A. M., \& Brady, P. M. (1987). Categorization of natural stimuli by monkeys (Macaca mulatta): Effects of stimulus set size and modification of exemplars. Journal of Experimental Psychology: Animal Behavior Processes, 13, 136-143.

Smith, E. E., \& Medin, D. L. (1981). Categories and concepts. Cambridge, MA: Harvard University Press.

Smith, J. E. K. (1982). Simple algorithms for M-alternative forcedchoice calculations. Perception \& Psychophysics, 31, 95-96.

Vonk, J., \& MCDonaLD, S. E. (2002). Natural concepts in a juvenile gorilla (Gorilla gorilla gorilla) at three levels of abstraction. Journal of the Experimental Analysis of Behavior, 78, 315-332.

Wasserman, E. A., DeVolder, C. L., \& Coppage, D. J. (1992). Nonsimilarity-based conceptualization in pigeons via secondary or mediated generalization. Psychological Science, $\underline{3}, \underline{374-378 .}$

(Manuscript received July 31, 2003; revision accepted for publication January 28,2004 .) 